\title{
A comparative Evaluation of Swarm Intelligence Algorithm Optimization: A Review
}

\author{
Shahab Wahhab Kareem ${ }^{1,2}$, Shavan Askar ${ }^{1}$, Roojwan Sc. Hawezi ${ }^{1}$, Glena Aziz Qadir ${ }^{1}$,Dina \\ Yousif Mikhail ${ }^{1,2}$ \\ ${ }^{1}$ Information System Engineering Department, Technical Engineering College, Erbil Polytechnic University, Erbil, Iraq \\ ${ }^{2}$ Department of Information Technology, Catholic University in Erbil, Kurdistan Iraq \\ Corresponding author: Shahab Wahhab Kareem (Shahab.kareem@epu.edu.iq).
}

\begin{abstract}
Swarm intelligence (SI), an important aspect of artificial intelligence, is increasingly gaining popularity as more and more high-complexity challenges necessitate solutions that are sub-optimal but still feasible in a fair amount of time. Artificial intelligence that mimics the collective behavior of a group of animals is known as swarm intelligence. Attempting to survive. Optimization contributes to optimal resource management by way of efficient and effective problem-solving. Engineers' attention has been driven to more effective and scalable metaheuristic algorithms as a result of the complicated optimization issues. It is primarily influenced by biological systems. The main aim of our article is to find out more about the guiding principle, classify possible implementation areas, and include a thorough analysis of several SI algorithms. Swarms can be observed in ant colonies, fish schools, bird flocks, among other fields. During this article, we will look at some Swarm instances and their behavior. The authors see many Swarm Intelligence systems, like Ant colony Optimization, which explains ant activity, nature, and how they conquer challenges; in birds, we see Particle Swarm Optimization is a swarm intelligence-based optimization technique, and how the locations must be positioned based on the three concepts. The Bee Colony Optimization follows, and explores the behavior of bees, their relationships, as well as movement and how they work in a swarm. This paper explores several algorithms such as ACO, PSO, GA, and FA.
\end{abstract}

INDEX TERMS Swarm Intelligence, Optimization, Particle Swarm Optimization, Ant Colony Optimization.

\section{INTRODUCTION}

Real optimization issues are difficult to be addressed by traditional means of computing since an intricate and complex nature of such problems may not guarantee an optimal solution [1]. The complexity of objective function and imposed constraints, mixed nature of variables and nonlinear relationships among variables has made the optimization in the field of engineering a challenging job for engineers. Therefore, a need to replace the complex techniques of hard computing with more intelligent processing techniques was felt [2]. SI is a creative distributed intelligence model for solving optimization problems that is inspired by biological scenarios such as swarming, flocking, and herding of vertebrates [3] [4] [5]. [6] developed the word Swarm Intelligence, which was motivated by "the emergent collective intelligence of clusters of basic agents"(Bonabeau, Marco, Dorigo, Théraulaz, \& Theraulaz, 1999) such as ants, bees, and bird flocks communicating among themselves and with their environment.

The collective intelligence possessed by these unsophisticated groups having limited capabilities of their own is attributed to their social interactions necessary for their survival. This coordinated and self-organized effort among the group decentralizes the task and guides the agents to their common goal through a coherent behaviour. The social behaviour exhibited by the simple agents or swarms scales down the most difficult task and has thus provided inspiration for the development of swarm intelligence-based optimization algorithms.

These nature-inspired algorithms have inherent robustness and versatility that enables them an effective architecture model for complicated engineering problems. Their success stems from their high productivity, which is due to information sharing among different participants, which 
contributes to self-organization, co-evolution, and understanding during iterations. Another factor is that several entities can be effectively parallelized, allowing large-scale optimization more realistic from an execution perspective [7]. Ant Colony and Particle Swarm Optimization originated from earlier developments in the field of swarm intelligence. Following this, some algorithms such as the Firefly Algorithm, Bat Algorithm, Artificial Bee Colony Algorithm, and many others appeared [8]. FIGURE 1 shows a description of optimization method. The research paper begins with a brief introduction that is section one, in section two implementations of several important swarm intelligence algorithms, including Particle Swarm Optimization, Firefly Algorithm, Ant Colony Optimization, Bat Algorithm, Artificial Bee Colony Algorithm, Cuckoo Search and Charged System Search. Section three swarm intelligence issues discussed. Section four, some literatures are reviewed then in section five they are discussed. And in section six the paper in concluded.

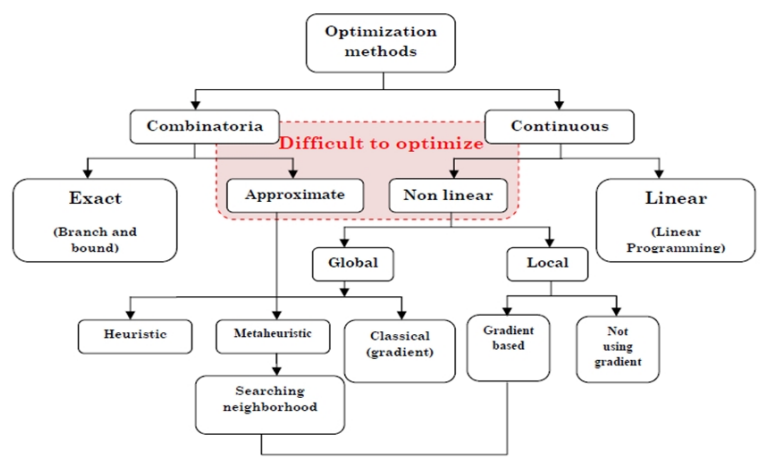

FIGURE 1. Classification of optimization methods addressed by [9].

\section{ALGORITHMS FOR SWARM INTELLIGENCE}

We will go throw the most common swarm algorithms in this section.

\section{A. PARTICLE SWARM OPTIMIZATION}

Kennedy and Eberhart developed the particle swarm optimization algorithm. That simulates flock social behaviour [10]. PSO simulates social behaviour among the group, by exploiting the tendency of individual to compete with the success of other individuals within the group. This is accomplished by sharing of knowledge or experience among the group individuals and therefore the success of an individual is governed by its own success and success of its neighbouring individuals. The hypothesis that a possible addressing to an optimization issue is considered as a bird without quality and size, called a particle, moving through a D-dimensional space, changing its location in search space based on its own skill and that of its neighbours, is the basis for the creation of PSO [11]. The quest for the best solution begins with a swarm, which is a set of random particles (or solutions). Each particle has a fitness function to be optimized. The fitness function creates a problem landscape in which each possible solution has a corresponding fitness value. Particles moving in this landscape adjust their position and velocity by exploiting their individual learning and cultural interactions to gather and share information regarding possible solutions to a problem thereby increasing their probability to arrive at an optimum solution. Searching for an optimal solution, the particles try to adapt to the dynamic environment through change in its three main properties: viz., inertia (attributed to its personal component), its best position (attributed to its personal experience) and the best position found by the group (attributed to social exchange of information among the group). PSO technique thus provides a balancing amalgam of exploration and exploitation through local search (through individual learning) and global search (through sharing of information with the neighbours) respectively [12].

\section{B. ANT COLONY OPTIMIZATION}

It is a meta-heuristic that is a group of artificial ants works together to solve difficult complex optimization issues. The ACO mechanism is designed to replicate the foraging characteristics of natural ant colonies, helping them for finding the shortest path to food that is shown in FIGURE 2. The collective behaviour among the individual ants is established through a chemical called pheromone, which is deposited at the food source. The quantity and consistency of the food supply determine the amount of chemical stored. The insects would use the pheromones to trace the path from their home to the source of food and likewise. The degree of probability to follow a route among various alternative routes increases with the majority of ants following a particular route. The pheromone trails act as simple indirect form of communication among these not so intelligent simple agents [13]. This cooperative behaviour is the key design component of ACO algorithm, which allows simple agents like artificial ants, to indirectly communicate and coordinate their activities through a mechanism called stemmery. This natural stemmery is transformed into a metaheuristic optimization known as ACO. In ACO, a group of insects find solutions to the issue at side and transmit information about the quality of such solutions using a cooperation scheme close to that used by real ants. The pheromone model is a parametrized probabilistic model that is at the core of an ACO algorithm (Dorigo \& Blum, 2005). The model parameters are pheromone trail associated to the components of solutions. The pheromone model adds probability to the solutions associated to a particular problem. It thus creates a probabilistic search space with numerous solutions. The ACO begins with a different 
value of ants and the initialization of the pheromone trail. The ants produce a complete solution to this issue by processing transactions through the dilemma landscape during each run of the ACO algorithm. After each ant has created a solution, the pheromone changing rule updates the power of artificial ants on chosen value [14]. This update, concentrates the global search for high quality solutions by increasing the bias towards sampling good solutions similar to those constructed in the past [15].

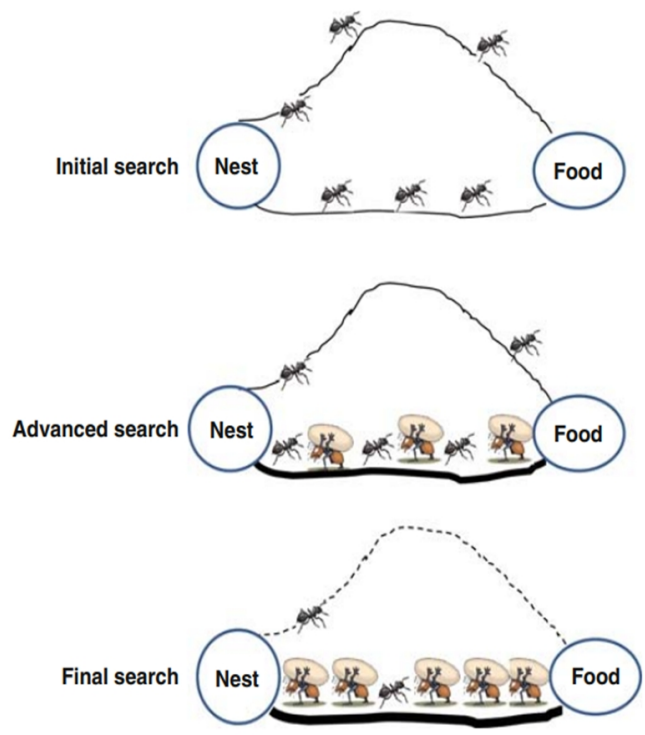

FIGURE 2. Ant route handling addressed by [16].

\section{FIREFLY ALGORITHM}

Xin-She Yang at Cambridge University created the Firefly Algorithm (FA) in 2007 [17], which was linked to the way butterflies attract mates by blinking their wings, possible prey, and function as a defensive alarm. It is a type of populationbased stochastic search algorithm motivated by the emitted light, absorption, and mutual attraction behaviours of fireflies. Since the blinking light's visibility is equal to its attraction, the brightness can be regarded as a goal feature that must be reduced or increased. The light of a maximization problem can be thought of as proportional to the optimal solution [18]. The FA begins with the population of fireflies being initialized, with each firefly representing a possible solution to the issue. The volume of the solution space is selected by the number of the population. The light intensity of each firefly or candidate solution is used to assess its health. The attraction of neighbouring fireflies is proportional to the amount of light they see, and its module can be described using light intensity, wavelength, and an absorption coefficient. The current location, beauty, and spontaneous walk of the firefly determine its movement. The brighter the firefly, the stronger its position and, more critically, the more powerful its appeal to other fireflies [19]. FA takes advantage of the light and appeal of fireflies to update its location in order to reach global maximum. This capacity splits the overall community into subgroups, for each group buzzing around a local optimum, allowing large populations to simultaneously discover local optima. The total distance a group of fireflies is seen by the neighbouring group is quantitatively controlled by the attraction among the fireflies. FA is especially well suited to strongly nonlinear, modality optimization problems because of this added benefit [20].

\section{BAT ALGORITHM}

Yang proposed the Bat Algorithm (BA) in 2010 [21], Echolocation features of microbats was the source of inspiration for this piece. Bats utilize echolocation to track food, clear obstacles, and find their nests in the darkness. In $\mathrm{BA}$, echolocation and frequency tuning is used to solve the problems. During flying, Bats have a random velocity and position with a fixed frequency. To spot the prey, various wavelengths and loudness are used [22]. They will automatically change the frequency and rate of pulses emitted depending on the target's proximity. A random vector is used to show the rate, real ideas, velocities at a given time phase, and a parameter used for this. The Bat Algorithm starts by creating a community of bats, each with a starting location, a pulse frequency, a loudness, and a find rate. The pulse rate and loudness are chosen at random. Through each iteration, each bat will progress from its original solution to the best global answer. If any bat seeks a better solution after jumping, the pulse emission and loudness are changed. The best answer so far is revised through flying iteration. This procedure is carried out indefinitely before the termination conditions are met. The optimal solution found so far is called the final perfect option [23].

\section{E. ALGORITHM FOR ARTIFICIAL BEE COLONY}

The $\mathrm{ABC}$ technique simulates a honey bee swarm's foraging behaviour. In 2005, Karaboga suggested it [24]. The ABC algorithm comprises of some groups of bees including worked bees, onlookers and scouts. Audience member refers to a bee that is staying on the main stage to make a decision about which food source to travel, whereas employed bee refers to a bee that is returning to a food source that it has already visited. The other kind of bee is the scout bee, which goes on a spontaneous hunt for new sources. The accuracy of response is related to the nectar volume of a base of food [25]. In the $\mathrm{ABC}$ system, the bees move around in a multi - dimensional searching, while others choose sources of food based on their and their nestmates' knowledge and change their places. Some (scouts) travel and select sources of food based on chance rather than skill. They remember the current location and ignore the previous one if the sum of nectar from a new source in their brain is greater than the previous one [26].

\section{F. CUCKOO SEARCH}


Cuckoo Search is designed by Yang and Deb, is one of the most recent evolution metaheuristic algorithms in 2009. CS is focused on certain cuckoo species' brood parasitism. Furthermore, the so-called Levy flights improve this algorithm. Instead of simple isotropic random walks According to recent research, CS has the ability to be much more effective than PSO and gas [27] [28].

\section{G. CHARGED SYSTEM SEARCH}

It is another metaheuristic algorithm that has only recently been implemented [29]. CSS algorithm is used to overcome a variety of optimization issues, including skeletal structure design, grillage device design [30], and MR damper parameter recognition, as well as the development of integrated open channels. The guiding laws of charged structures inspired this algorithm. CSS uses several agents/charged particles, similar to swarm algorithms [31]. Because they are viewed as CP, these entities can interact with one another using the Gauss electrostatics principles. The Newtonian provides the managing principles of motion. Social services can impose electrical forces on each other, and the stresses vary depending on the potential difference between the cps and are proportional to the frequency of the potential difference between the particles with a $\mathrm{CP}$ outside the sphere. Each iteration, each one of cps move to its newer position, taking into account the resulting abilities and its previous velocity. When a CP leaves the allowable search space, Kaveh and Talatahari's HS-based management technique is used to determine its position [30].

\section{ISSUES OF SWARM INTELLIGENCE}

There are two major algorithmic shortcomings in PSO. The first downside is that PSO is vulnerable to premature convergence when addressing complicated problem optima. Since the original PSO isn't a regional optimizer, the resulting solution can't be guaranteed to be a local optimum. [32]. PSO's performance is strongly influenced by parameter settings, which is one of its flaws. Increasing particle speed by increasing the inertia weight, for instance, allows for more exploration and less manipulation, and so on. The method for correcting inertia varies depending on the problem ( [33]. Aside from such technical problems, the ACO and PSO lack a strong mathematical basis for research, particularly in terms of operational algorithm convergence conditions and simple parameter estimation methodologies. There are some doubts regarding swarm intelligence's "dark side." Opposition to two future swarm intelligence implementation areas, army management and biomedical, is rising on the social front. In the war against terrorism, the first domain region has always gotten a lot of coverage. As a consequence, planning and hiring driving and combat operations is no longer statistically significant. It has the potential to transform the way war and intelligence are conducted in the near future. The idea of a technological Big Brother watching and creeping on intelligent swarms, on the other hand, is disturbing. [34]

\section{LITERATURE REVIEW}

In the optimization environment, swarm intelligence is a hot topic. PSO, ACO, ABC, BA, and firefly algorithm are only a few of the effective optimization algorithms inspired by swarm intelligence that have been developed in recent years [35]. We will summarize the relevant work in the area of Swarm Intelligence algorithms in this section.

[36] Proposed a real-time filtering and noise reduction paradigm for nonlinear vibration signals. By adding a mutation operator, this algorithm solves the problem where the PSO approach easily sinks into local ideal value with low measurement precision around the same time, the mutation operation increases the sampling process' particle distribution and variety. The issue with the particle filter (PF) method, at which samples are poor and the utilization rate is low, has been resolved as well.

To take into account all stakeholders, a three-objective gate allocation model is used, in which the shortest passenger walking distances, the most balanced idle period of each gate, and the fullest use of big gates are all configured at the same time to maximize functional performance. To efficiently solve this model, [37] proposed a model. They validated the feasibility of the suggested approach using data from an Airport's real operations.

U-type assembly lines are extensively applied in modern manufacturing systems for higher flexibility and productivity. [38] Presented a model to decrease the number of stations. Rather than getting just one task assignment at a time, this approach produces a series of task assignments and chooses the right one for the current station. The suggested strategy is used to solve a series of benchmark problems, and the results are compared to those obtained by state-of-the-art approaches (such as ULINO) and variations of the ant colony optimization technique.

Marine services are critical to a country's growth. A traditional procedure used in marine investigations takes a long time and costs a lot of money. As a result, scientific route forecasting is critical for increasing the productivity and lowering the costs of marine research. The main findings achieved by GA and ACOA eventually reach the optimum solutions by continuous iterations. As a result, [39] suggested a method for more improving the accuracy of the approaches to address these issues and save costs associated with marine research.

A social network's group structure is an essential component. In a wide variety, of applications identifying certain groups is critical. Finding communities is a difficult challenge. [40] Suggested the Clustering Coefficient-based Genetic Algorithm (CC-GA) for finding them in complicated social networks. 
Both ANFIS have shown to be useful tools for predicting engineering issues. [41] Used GA and PSO to develop ANFIS. The full-scale effects of the available supervised piles were used to assemble the training data list. The ANFIS model was learned in MATLAB using a learning fuzzy-based technique. The system was set up by changing the size of groups in the FIS, then running the GA and PSO optimization algorithms on the output.

[42] Suggested a new dynamic Firefly algorithm for request analysis of water supply in Nanchang, China. To stop manually changing the phase component, a complex parameter approach is utilized first. Second, in terms of historical water usage and local economic structure, three estimation models in various forms are created. Third, the normalization process is used to remove the effects of various data units.

\section{METHODOLOGY}

The majority of Swarm Intelligence algorithms were developed to overcome real-world optimization problems in the solution space. Some Swarm intelligence algorithms in various applications and conditions are studied and compared in this section. The (NPSO-PF) is a new particle swarm optimization particle filter algorithm. It is developed to filter and reduce noise in nonlinear vibration signals in live time. The problem with the PF method where the samples are weak and the usage amount is low is fixed.

The quantum evolutionary algorithm (QEA) is a quantum computing and EA-based probabilistic searching algorithm. It has a stronger population dispersion, a faster convergence speed, a powerful search capability, and is simple to integrate with other algorithms. The improved QEA, named IPOQEA, is based on the niche co-evolution technique and enhanced particle swarm optimization (PSO). To validate the effectiveness of the proposed method, 250 flights and 30 gates are used from Guangzhou Baiyun International Airport. To assign flights to appropriate gates within various times, an IPOQEA-based gate allocation system is proposed. Furthermore, the IPOQEA has superior optimization capabilities when it comes to addressing gate allocation problems. As a result, the current gate allocation system has a lot of scope for functional engineering because it allows airport managers to make quick decisions.

ACO is an important tool for addressing complicated engineering architecture issues that has already been commonly utilized. Recently, this optimization technique has been used to address a variety of assembly line balance issues. To reduce the number of stations, a new mixed-integer linear programming (MILP) paradigm is proposed. The stationoriented protocol significantly increases the efficiency of the initial ant colony optimization. The computational study demonstrates the superiority of the proposed method over the compared ones as it achieves optimal solutions for 255 cases (out of 269) and outperforms the current best method, ULINO, for 21 cases. In brief, this station-oriented procedure generates a set of possible task assignments (or groups of tasks), and then selects the best one as the current task assignment. The illustrated case has 70 tasks and the cycle time is fixed to 527 units. Supposed that the number of task assignment or $\mathrm{LN}$ is set to 10 .

GA is a search-driven optimization method depending on genetics and natural selection concepts. It's often employed to discover ideal or near-optimal approaches to complex issues that would take a lifetime to address anyway. It's broadly utilized in machine learning to address optimization difficulties. The ACO and GA are merged to suggest a hybrid model for further improving the efficiency of approaches, which has been shown to be highly strong and reliable. Due to its excellent performance, the ACOA is often applied to solve the traveling salesman problems; currently, lots of the ideas of solving traveling salesman problems depend on the ACOA.

The Clustering Coefficient-based Genetic Algorithm (CCGA) is proposed for defining populations in complex networks. Designers have utilized a variety of genetic algorithms to identify populations, but the suggested technique is unique in terms of both the initial population generation and the mutation process, both of which increase the performance and accuracy of the system. They evaluated the performance of CC-GA with 11 different real-world networks. The networks belong to four categories: social; biological; collaborative; 350 and online-social. The size of these networks ranges from tens up to tens of thousands of nodes. The main objective of selecting these different types of networks of various sizes is to test the performance and scalability of the proposed algorithm on dissimilar networks. A non-linear adaptive framework is the Adaptive-networkbased fuzzy inference system (ANFIS). For the measurement of the friction power ratio $(\alpha)$ in powered shafts, improving ANFIS with GA to present GA-ANFIS and ANFIS with PSO to introduce PSO-ANFIS. Provided the database utilized to create both ANN and ANFIS models in this work. The initial data comes from a full-scale research of non-displacement pile base resistance in the sand. The most important parameters are pile diameter $(\mathrm{m})$, pile length $(\mathrm{m})$, relative density, and CPT qc $(\mathrm{MPa})$, with friction being the primary output.

The Firefly algorithm (FA) is a swarm intelligence-based optimization technique that has been effectively implemented to a variety of engineering issues In Nanchang, China, a modern approach FA called NDFA is suggested for requesting analysis of water supply, with a prediction accuracy of up to 97.91 percent.

The authors demonstrate how FA can be used to estimate water demand in Nanchang, China. Water demand is influenced by social and economic factors. From 2003 to 2015, Table 1 displays the historical water use in Nanchang. It can be seen that Nanchang's water use is divided into three categories: agricultural, industry, and inhabitants. 


\section{DISCUSSION AND RESULT}

Because of the huge success of Swarm Intelligence algorithms in addressing a wide range of optimization problems effectively. As seen in Table 1, each Swarm Intelligence algorithm used during analysis of this paper is outlined in this section based on the methods and findings of every model. Experiments have shown that NPSO-PF has lower root mean square error, shorter running time, and higher signal to noise ratio than the alternative (PF) algorithms and the NPSO-PF method.

The algorithm uses a number of genetic algorithms (algorithms that generate new solutions from pre-existing solutions) but does so in a unique way, which increases the algorithm's effectiveness and accuracy [43]. An in-depth study of real-world datasets, including simulated datasets, finds that experiments yield better outcomes than current genetic and non-genetic algorithms.

Based on computational analysis, it appears that all five of the FA forms have good results. Using an NDFA model, it is found that it provides superior performance than the four other FA types, and the accuracy of its predictions is found to be up to 97.91 percent. Lastly, the city of Nanchang's water consumption is estimated during the next five years.

The flights are suggested to be allocated to different gates by using an IPOQEA-based gate allocation mechanism. Finally, the real data used to evaluate the efficacy of the suggested method is from Baiyun Airport. The results demonstrate that the model designed in this study can meet all three of these characteristics of airport management. Additionally, the IPOQEA has stronger gate allocation optimization abilities.

In this computational analysis, the new technique exceeds the existing best method, ULINO, for 21 situations and is superior to it for 255 cases (out of 269). Also noteworthy is the fact that by employing a station-oriented technique, original ant colony optimization delivers better results.

The ACO and GA have blended their algorithm into a hybrid approach that can enhance solutions quality. In order to determine the ideal path for single or several research vessels, the suggested hybrid algorithm is demonstrated to be both very effective and robust, as demonstrated by the successful experiments and the software implementation.

\section{CONCLUSIONS}

There are a multitude of uses for the term "optimization." Optimization contributes to good resource management through efficient and effective problem solving. Traditional computing may not be able to achieve optimal solutions due to the complicated and complex nature of these challenges. Due to the complexity of optimization difficulties that engineers have to face, the focus of engineers has been directed toward more effective and scalable metaheuristic algorithms. The metaheuristic optimization field that has great potential is the swarm intelligence. The Swarm Intelligence methods implemented use a mixture of stochastic, populationbased metaheuristics, which draw inspiration from a variety of natural events. The process of socialization for ants has been inspirational for bee swarms, wasp flocks, and other bird flocks, who naturally tend to organize themselves to optimize their work. Swarm-based optimization algorithms look to the cooperative behaviour of these simpler agents and encourage self-organization. Additionally, iterations provide an opportunity for learning. A variety of optimization algorithms were demonstrated in the review article, some of which use swarm intelligence. When dealing with huge optimization issues, these simple agents perform their computations faster because they are similar to one another.

\section{REFERENCES}

[1] Abraham, A., Guo, H., \& Liu, H. (2006). "Swarm intelligence: foundations, perspectives and applications". In "Swarm intelligent systems": Berlin, Heidelberg, Springer, 2006, pp. 3-25)

[2] Aydoğdu, ., Akın, A., \& Saka, M. "Discrete design optimization of space steel frames using the adaptive firefly algorithm", Paper presented at the The 11th International Conference on Computational Structures Technology, Dubrovnik, Crotia, 2012, pp 1168-1184.

[3] Beni G., Wang J. "Swarm Intelligence in Cellular Robotic Systems", In "Robots and Biological Systems: Towards a New Bionics?". NATO ASI Series (Series F: Computer and Systems Sciences), vol 102. Springer, Berlin, Heidelberg, 1993, (pp. 703-712).

[4] Eric Bonabeau, Marco Dorigo, Guy Theraulaz, "Swarm intelligence: from natural to artificial systems", 1st ed. Oxford university press, USA, 1999.

[5] Camp, C. V., Meyer, B. J., \& Palazolo, P. J. "Particle swarm optimization for the design of trusses". In Structures Congress, Nashville, Tennessee, United States, 2004 (pp. 1-10).

[6] S. W. Kareem, Novel Swarm Intelligence Algorithms For Structure Learning Of Bayesian Networks And A Comparative Evaluation, Ph.D dissertation, Graduate School Of Natural And Applied Sciences, Yasar university, 2020.

[7] Carbas S, Hasancebi O. "Optimum design of steel space frames via bat inspired algorithm". In: 10th World congress on structural and multidisciplinary optimization; At: Orlando, Florida, USA, 2013, (pp. 1-10).

[8] Hanxin Chen, Dong Liang Fan, Lu Fang, Wenjian Huang, Jinmin Huang, Chenghao Cao, Liu Yang, Yibin He and Li Zeng, "Particle swarm optimization algorithm with mutation operator for particle filter noise reduction in mechanical fault diagnosis", in International Journal of Pattern Recognition Artificial Intelligence. vol.34, no.10, 2020.

[9] Chen, J., Su, M., Shen, S., Xiong, H., \& Zheng, H. "POBA-GA: Perturbation optimized black-box adversarial attacks via genetic algorithm". Computers Security, vol. 85, 2019 (pp.89-106).

[10] Deng, W., Xu, J., Zhao, H., \& Song, Y. "A Novel Gate Resource Allocation Method Using Improved PSO-Based QEA". IEEE Transactions on Intelligent Transportation Systems, 2020 (pp. 1-9).

[11] Deng, W., Yao, R., Zhao, H., Yang, X., \& Li, G. J. S. C. (2019). A novel intelligent diagnosis method using optimal LS-SVM with improved PSO algorithm. vol. 23, no.7, 2019 (pp.2445-2462).

[12] M. Dorigo, M. Birattari and T. Stutzle, "Ant colony optimization," in IEEE Computational Intelligence Magazine, vol.1, no.4, 2006 (pp.28-39), doi: 10.1109/MCI.2006.329691.

[13] S. Kareem and M. Okur, "Bayesian network structure learning based on pigeon inspired optimization,", International Journal of Advanced Trends in Computer Science and Engineering, Vol. 8, no.1.2 2019, (PP.131-137). 
[14] Engelbrecht, A. P. "Computational intelligence: an introduction", 2nd ed., Chichester, West Sussex, England. John Wiley \& Sons, 2007.

[15] Fister, I., Jr.; Yang, X.S.; Brest, J.; Fister, D. "A Brief Review of Nature-Inspired Algorithms for Optimization". Elektrotehniški Vestn, Vol. 80, no.3, 2013 (pp. 116-122).

[16] Amir Hossein Gandomi,Siamak Talatahari,Xin-She Yang,Suash Deb, " Design optimization of truss structures using cuckoo search algorithm", in Struct. Design Tall Spec. Build. vol. 22, no.17, 2012, (pp. 1330-1349).

[17] Gandomi, A. H., Yang, X.-S., \& Alavi, A. H. "Mixed variable structural optimization using frefy algorithm", in Computers and Structures, vol.89, no. (23-24), 2011, (pp. 2325-2336).

[18] M. Ghazavi, S. Bazzazian Bonab, "Optimization of reinforced concrete retaining walls using ant colony method", ISGSR - Vogt, Schuppener, Straub \& Bräu (eds), 2011, (pp. 297-305).

[19] O. Hasançebi, T. Teke, O. Pekcan, "A bat-inspired algorithm for structural optimization", Computers \& Structures,vol. 128, no.11, 2013, (pp. 77-90).

[20] M. Janga Reddy,D. Nagesh Kumar "Evolutionary algorithms, swarm intelligence methods, and their applications in water resources engineering: a state-of-the-art review", H2Open Journal, vol.3, no.1, 2020, (pp.135-188)

[21] Karaboga, D., \& Celal Öztürk, "A novel clustering approach: Artificial Bee Colony (ABC) algorithm", Appl. Soft Comput, vol. 11, no.1, 2011, (pp. 652-657).

[22] Ali Kaveh and Siamak Talatahari and Bahman F. Azar, "Optimum design of composite open channels using charged system search algorithm", Journal of Science and Technology. Transactions of Civil Engineering, vol. 36, no.1, 2012, (pp.67-77).

[23] S. W. Kareem, R. Z. Yousif, and S. M. J. Abdalwahid, "An approach for enhancing data confidentiality in hadoop," Indonesian Journal of Electrical Engineering, vol. 20, no. 3, 2020, (pp. 1547-1555).

[24] Kaveh, A., \& Talatahari, S. J. J. o. C. S. R. (2010). "Charged system search for optimum grillage system design using the LRFD-AISC code", Journal of Constructional Steel Research, vol. 66, no. 6, 2010 (pp. 767-771)

[25] S. M. J. Abdalwahid, R. Z. Yousif, and S. W. Kareem, "Enhancing approach using hybrid pailler and RSA for information security in bigdata," Applied Computer Science, vol. 15, no. 4, 2019 (pp. 6374).

[26] S. W. KAREEM and Mathematics, "Secure Cloud Approach Based on Okamoto-Uchiyama Cryptosystem," Journal of Applied Computer Science, vol. 14, no. 29, 2020, (pp. 9-13).

[27] Jun-qing Li, Quan-ke Pan, " Solving the large-scale hybrid flow shop scheduling problem with limited buffers by a hybrid artificial bee colony algorithm", Information Sciences, vol. 316, 2015, (pp.487502).

[28] S. Kareem and M. C. Okur, "Bayesian Network Structure Learning Using Hybrid Bee Optimization and Greedy Search," 3rd International Mediterranean SCIENCE AND ENGINEERING CONGRESS, At: Adana-turkey, 2018.

[29] Liang, Y., \& Wang, L. "Applying genetic algorithm and ant colony optimization algorithm into marine investigation path planning model". Soft Computing, vol. 24, no. 11, 2020, (pp. 8199-8210).

[30] Marini, F., Walczak, B. J. C., \& Systems, I. L. "Particle swarm optimization (PSO). A tutorial". Chemometrics and Intelligent Laboratory Systems, vol. 149, 2015, (pp.153-165).

[31] S. W. Kareem and M. C. Okur, "Evaluation of Bayesian Network Structure Learning Using Elephant Swarm Water Search Algorithm," in Handbook of Research on Advancements of Swarm Intelligence Algorithms for Solving Real-World Problems: IGI Global, 2020, (pp. 139-159).

[32] S. W. Kareem and M. C. Okur, "Structure learning of Bayesian networks using elephant swarm water search algorithm," International Journal of Swarm Intelligence Research, vol. 11, no. 2, 2020, (pp. 19-30).

[33] B. Niu, Y. Zhu, X. He and H. Wu, "MCPSO: A multi-swarm cooperative particle swarm optimizer," Applied Mathematics and Computation, Vol. 185, 2007, (pp. 1050-1062).
[34] N. Priyadarshi, S. Padmanaban, J. B. Holm-Nielsen, F. Blaabjerg and M. S. Bhaskar, "An Experimental Estimation of Hybrid ANFISPSO-Based MPPT for PV Grid Integration Under Fluctuating Sun Irradiance," in IEEE Systems Journal, vol. 14, no. 1, pp. 1218-1229, March 2020, doi: 10.1109/JSYST.2019.2949083.

[35] Said, A., Abbasi, R. A., Maqbool, O., Daud, A., \& Aljohani, N. R. (2018). "CC-GA: A clustering coefficient based genetic algorithm for detecting communities in social networks". Applied Soft Computing, vol. 63, 2018, (pp. 59-70).

[36] S. Kareem and M. C. Okur, "Evaluation Of Bayesian Network Structure Learning," in 2nd International Mediterranean Science and Engineering Congress (IMSEC 2017), Adana, TURKEY, 2017.

[37] Talatahari, S., Mohaggeg, H., Najafi, K., \& Manafzadeh, A. J. M. P. i. E. "Solving parameter identification of nonlinear problems by artificial bee colony algorithm", Mathematical Problems in Engineering, 2014.

[38] S. W. Kareem and M. C. Okur, "Pigeon inspired optimization of bayesian network structure learning and a comparative evaluation," Journal of Cognitive Science, vol. 20, no. 4, 2019, (pp. 535-552).

[39] Varaee, H., \& Ahmadi-Nedushan, B. J. W. A. S. J. (2011) "Minimum cost design of concrete slabs using particle swarm optimization with time varying acceleration coefficients", World Applied Sciences Journal, vol. 13, no.12, 2011, (pp. 2484-2494).

[40] R. Z. Yousif, S. W. Kareem, and S. M. Abdalwahid, "Enhancing Approach for Information Security in Hadoop," Polytechnic Journal, vol. 10, no. 1, 2020, (pp. 81-87).

[41] X. S. Yang, Nature-Inspired Metaheuristic Algorithms, Luniver, Frome, UK, 2008.

[42] Yang, X.-S., \& Deb, S. "Cuckoo search via Lévy flights". Paper presented at the 2009 World congress on nature \& biologically inspired computing (NaBIC), 2009.

[43] Shavan Askar, Zhala Jameel Hamad, Shahab Wahhab Kareem. Deep Learning and Fog Computing: A Review. International Journal of Science and Business, Vol. 5, no. 6, 2021, (pp. 197-208). doi https://doi.org/10.5281/zenodo.5222647.

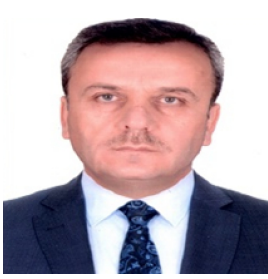

Dr. Shahab Wahhab Kareem Shahab Wahhab Kareem, Shahab Wahhab Kareem his BSc in Control and Computer Engineering from the University of Technology Baghdad in 2001, MSc in Software Engineering from Salahadeen University in 2009, and $\mathrm{Ph} . \mathrm{D}$. in Yasar University Izmir, Turkey in 2020. My research interests include Machine learning and BIG DATA. I'm a lecturer at the Information System Eng. (ISE) Department (2011-till now).

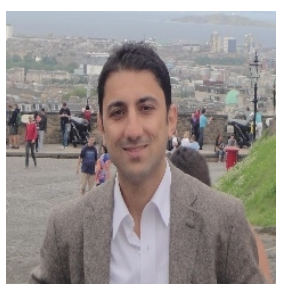

Dr. Shavan Askar (Associate Professor of Computer Networks) is currently faculty member at Erbil Polytechnic UniversitylCollege of Engineering. He received his $\mathrm{PhD}$ degree in Electronic Systems Engineering from the University of EssexlUK in 2012. He obtained his MSc (2003) and BSc (2001, Ranked 1st on the college) degrees from the Control and Systems Engineering Dept. Baghdad. Dr. Askar works in the field of Networks that includes Internet of Things, Software Defined Networks, Optical Networks, and 5G. Dr. Askar has started his academic career in 2003 when he was appointed as a lecturer at the University of Duhok until 2008 when he was granted a scholarship to do his PhD degree that commenced in October 2008 and finished successfully in June 2012. Dr. Askar then returned to Iraq to pursue his academic career at the University of Duhok for the period 2012 2016 by supervising master students, teaching post-gradatue courses, and became project manager of so many strategic projects in Kurdistan. In 2016, Dr. Askar joined Duhok Polytechnic University as the Director General of Scientific Research Center, his role includes apart from teaching post-graduate students, contributing to 
the development of the university from the technological and scientific perspectives. After 2017, Dr. Askar has started working, beside to his public university tasks, as an Adjunct Professor at the American University of Kurdistan, he contributed into the establishment of a new program called Electronic and Telecommunications Engineering $\backslash$ College of Engineering, he taught different courses in this program.

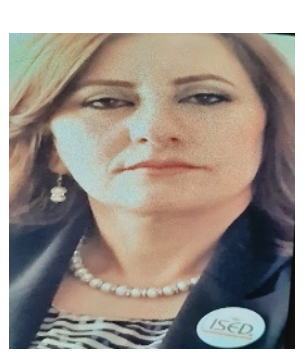

\section{Dr.Roojwan Scddeek Hawezi}

I got the BSc degree from Baghdad University in Physical science, then I got a higher Diploma in Computer science. Back in 2003 I got my MSc degree then finally the $\mathrm{PhD}$ in 2007 in Computer science from Baghdad too. I'm a specialist at Digital Image Processing. I'm the former head of the Information System Eng. (ISE) Department

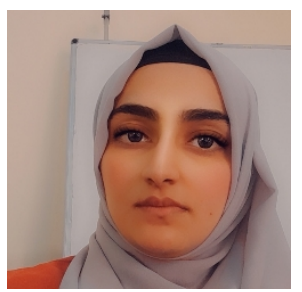

Glena Aziz Qadir, BSc in Information System Engineering from Erbil polytechnic university in 2017, MSc student in Information System Engineering from Erbil polytechnic university. I'm a team leader at Tech eye Company.

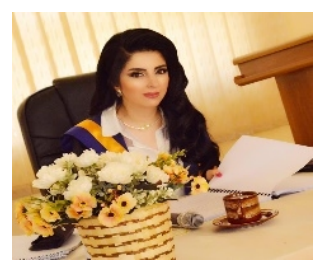

Dina Yousif Mikhail: Obtained B.Sc. (Bachelor of engineering) Medical Instrumentation engineering at 2003/2004 in Technical Collage University of Mosul-Iraq and Higher Diploma (Software Engineering) at 2009/2010 in Engineer CollageUniversity of Sallah Al Dien, ErbilIraq. She got M.Sc. degree in Engineering Technical Collage - Erbil, Polytechnic University Erbil-Iraq. She is currently in the department of Information Systems Engineering, Technical Engineering College, Erbil Polytechnic University, IRAQ. Her research interests in web application, bioinformatics \& biomedical Engineering, Artificial intelligent. She has 10 -year experience in teaching an undergraduate in information systems engineering. 